PUPT-2085

\title{
D-brane Decay in Two-Dimensional String Theory
}

\author{
Igor R. Klebanov, 1 Juan Maldacena and Nathan Seiberg \\ Institute for Advanced Study \\ Princeton, New Jersey, 08540, USA
}

\begin{abstract}
We consider unstable D0-branes of two dimensional string theory, described by the boundary state of Zamolodchikov and Zamolodchikov hep-th/0101152 multiplied by the Neumann boundary state for the time coordinate $t$. In the dual description in terms of the $c=1$ matrix model, this D0-brane is described by a matrix eigenvalue on top of the upside down harmonic oscillator potential. As suggested by McGreevy and Verlinde hepth/0304224, an eigenvalue rolling down the potential describes D-brane decay. As the eigenvalue moves down the potential to the asymptotic region it can be described as a free relativistic fermion. Bosonizing this fermion we get a description of the state in terms of a coherent state of the tachyon field in the asymptotic region, up to a non-local linear field redefinition by an energy-dependent phase. This coherent state agrees with the exponential of the closed string one-point function on a disk with Sen's marginal boundary interaction for $t$ which describes D0-brane decay.
\end{abstract}

May 2003

1 On leave from Physics Department, Princeton University. 


\section{Introduction}

In string theory there are various known cases of unstable D-branes containing open string tachyon modes on their world volume; for example, the D-branes of 26-dimensional bosonic string. In a series of seminal papers Sen proposed a boundary conformal field theory (BCFT) description of the decay of unstable D-branes [1]- 3 . In the bosonic string theory, the BCFT contains a boundary interaction

$$
\lambda \int d \tau \cosh \left[X^{0}(\tau) / \sqrt{\alpha^{\prime}}\right]
$$

whose exact marginality may be argued via continuation from the conformal invariance [4,5] of the Euclidean boundary Sine-Gordon theory. A more detailed study of D p-brane decay was carried out in [6-24] and led to many new insights. A number of puzzles remain however; for example, to leading order in $g_{s}$ the number of closed string produced in D0-brane decay is UV divergent and one needs to go beyond tree level for handling this divergence.

In a thought-provoking recent paper [25], a new laboratory for resolving these puzzles was proposed by J. McGreevy and H. Verlinde, the 2-dimensional bosonic string theory (for reviews, see 26-28). In $D=2$, the closed string tachyon becomes massless, so that the theory has no perturbative instability. The theory is not translation-invariant in the "Liouville direction" $\phi$, with the string coupling varying as $g_{s}(\phi) \sim e^{2 \phi / \sqrt{\alpha^{\prime}}}$. The 2-d string theory was explored intensively in the late 80's and early 90's due in part to its dual formulation in terms of quantum mechanics of a large $N$ hermitian matrix often referred to as the $c=1$ matrix model. The exact solvability of this large $N$ model (it reduces to $N$ matrix eigenvalues that behave as free fermions [29]) yielded a wealth of perturbative information about the theory, some of which has been reproduced using Liouville theory methods. Furthermore, the model has provided an important hint [30] that stringy nonperturbative effects are of order $e^{-A / g_{s}}$. From the point of view of the matrix model, such effects are due to single eigenvalue tunnelling [30] (see also [31]). This observation preceded the understanding that in string theories such $O\left(e^{-A / g_{s}}\right)$ effects are provided by D-instantons [32,33]. A more general connection between single eigenvalues in the matrix model and D-branes in the 2-d string theory was recently proposed in [25]. These authors examined a single eigenvalue balancing at the top of the upside down harmonic oscillator potential, and studied its decay by rolling down the potential. 
There has also been important recent progress in understanding D-branes of Liouville theory. In fact, as we review in the next section, this theory contains different types of D-branes [34 36]. The D-branes studied in [34,35] (and [25]) are extended in the Liouville direction and, after inclusion of the time direction, should be thought of as D1-branes. They are stable. There are also unstable D-branes localized at large $\phi$ that were discovered and studied by A.B. and Al.B. Zamolodchikov [36]. After inclusion of the time direction of the 2-d string, these are D0-branes. They are remarkable in that the open string theory that lives on them is exactly equivalent to quantum mechanics of a tachyon mode (the only oscillator excitation of the open string gives a non-dynamical gauge field which implements a constraint on the wave functions). The importance of the localized D-branes of [36] was emphasized and the question of their matrix model description was raised in [37].

We suggest that the unstable single eigenvalue of [25] should be identified with the unstable D0-brane of [36]. This D-sphaleron picture for the D0-brane fits nicely with the single-eigenvalue tunnelling description of the D-instanton anticipated in [30,32], and also with the general D-sphaleron interpretation of unstable D-branes in [38](see also [39]).

More generally, we suggest that the D0-branes dual to the matrix eigenvalues are the unstable D0-branes of [36]. This provides an exact duality between open strings on D-branes and closed strings [25].

In the present paper we calculate the decay amplitude of a D0-brane into closed strings. We show that, to leading order in $g_{s}$ the matrix model result agrees with that in Liouville theory (our results, both in the matrix model and in Liouville theory, differ from those in [25]). This leading order rate diverges in the UV in the same way as in the

higher-dimensional calculation of [23], but the matrix model approach demonstrates how to regulate this divergence. In the matrix model the precise regularization depends on the initial wavefunction we choose for the eigenvalue. Thus, the matrix model once again seems to provide important intuition about the quantum behavior of string theory.

\section{D-branes in $2 d$ string theory}

In this section we discuss the different D-branes that exist in two dimensional string theory. 
We are interested in a $2 \mathrm{~d}$ CFT which is the product of standard flat time direction and Liouville theory with $c_{L}=25$. Let us first collect formulae and conventions about Liouville theory.2

$$
c_{L}=1+6 Q^{2}, \quad Q=b+1 / b, \quad \Delta=\frac{1}{4} \alpha(2 Q-\alpha), \quad V=e^{\alpha \phi / \sqrt{\alpha^{\prime}}}
$$

For $b=1$, we have $Q=2, c_{L}=25$. The delta function normalizable states have

$$
\alpha=Q+i \sqrt{\alpha^{\prime}} P, \quad \Delta=\frac{Q^{2}}{4}+\frac{\alpha^{\prime}}{4} P^{2}
$$

with real $P$.

When $b \rightarrow 1$ the amplitudes in Liouville theory diverge. In order to obtain finite amplitudes we must also send $\mu_{0} \rightarrow \infty$ keeping

$$
\mu \equiv \pi \mu_{0} \gamma\left(b^{2}\right)=\text { finite }
$$

where $\gamma\left(b^{2}\right)=\Gamma\left(b^{2}\right) / \Gamma\left(1-b^{2}\right)$. The dependence on $\mu_{0}$ of the Liouville expressions in [36], [34, is only in the combination that remains finite as in (2.3), so that we obtain finite expressions. The parameter $\mu$ will be identified with the matrix model fermi energy.

\subsection{Extended D-branes in Liouville theory - D1-branes}

There are two types of D-branes that have been described in Liouville theory. First we have D-branes which are extended along the Liouville direction that were studied in [34, 35, 40, For these D-branes we can add a boundary cosmological constant $\mu_{B}$. The one-point function for a bulk operator $e^{\alpha \phi / \sqrt{\alpha^{\prime}}}$ is given by

$$
U_{\alpha}=\left(\pi \mu_{0} \gamma\left(b^{2}\right)\right)^{-\frac{i \sqrt{\alpha^{\prime}} P}{2 b}} \Gamma\left(1+i \sqrt{\alpha^{\prime}} b P\right) \Gamma\left(1+i \sqrt{\alpha^{\prime}} P / b\right) \frac{2 \cos \left(\pi s \sqrt{\alpha^{\prime}} P\right)}{i \sqrt{\alpha^{\prime}} P}
$$

where $s$ is a parameter parameterizing the boundary state related to the boundary cosmological constant by

$$
\cosh ^{2} \pi s b=\frac{\mu_{0, B}^{2}}{\mu_{0}} \sin \pi b^{2}
$$

Note that $s$ could be real or imaginary depending of whether the right hand side of (2.5) is bigger or smaller than one.

2 Our conventions are related to the ones in [34,36] by $\alpha^{\text {here }}=2 \alpha^{\text {there }}, \sqrt{\alpha^{\prime}} P^{\text {here }}=2 P^{\text {there }}$. 
These are the formulae for general $b$. As we remarked above the formulae for $b=1$ can be obtained by taking the limit (2.3). In this case it is also useful to rescale $\mu_{0, B}$ in the same way as $\mu_{0}$ (as in (2.3)). In that way we obtain a finite expression in the right hand side of (2.5) for $b=1$. So we conclude that also for $b=1$ we have a continuous family of boundary states parametrized by $s$. All D-branes in this family have a continuous spectrum of open string states; they are the open string "tachyons" which are massless (as are the closed string "tachyons"). In particular we can set this rescaled $\mu_{B}=0$. This gives us $s=i / 2$. This is just one particular D-brane state out of this continuous family. This is the D-brane considered by [25].

For $b=1$, the one point function (2.4) exhibits poles at imaginary values of $P=$ $i n / \sqrt{\alpha^{\prime}}$ with integer $n$. The meaning of these poles is well understood in the matrix model literature [26,27]. For such values of $P$ the $\phi$ charge of $U_{\alpha}$ can be screened with an integer number of insertions of the worldsheet cosmological constant. Then the correlation function diverges due to the volume of the noncompact $\phi$ direction. These divergences signal the fact that the correlation function is dominated by the bulk of space time - the region $\phi \rightarrow-\infty$. Therefore these poles signal that these D-branes are extended in the $\phi$ direction.

\subsection{Localized D-branes in Liouville - D0-branes}

There is a second class of Liouville theory D-branes that were considered in [36]. These D-branes should be thought of as localized in the Liouville direction at $\phi=\infty$, which is the strong coupling end. The localized D-branes are parametrized by two integers $(n, m)$. We are interested in the case $(n, m)=(1,1)$. The other ones contain operators more tachyonic than the tachyon in the open string spectrum. It is not clear what they correspond to.

The bulk one point function in this case reads (eq. (5.10) of [36])

$$
\mathcal{A}_{L} \sim\left(\pi \mu_{0} \gamma\left(b^{2}\right)\right)^{-i \frac{\sqrt{\alpha^{\prime}} P}{2 b}} \frac{\pi i \sqrt{\alpha^{\prime}} P}{\Gamma\left(1-i \sqrt{\alpha^{\prime}} b P\right) \Gamma\left(1-i \sqrt{\alpha^{\prime}} P / b\right)}
$$

Taking the $b=1$ limit as described above we get a simple finite answer

$$
\mathcal{A}_{L}=\frac{2}{\sqrt{\pi}} i \sinh \left(\pi \sqrt{\alpha^{\prime}} P\right) \mu^{-i \frac{\sqrt{\alpha^{\prime}} P}{2}} \frac{\Gamma\left(i \sqrt{\alpha^{\prime}} P\right)}{\Gamma\left(-i \sqrt{\alpha^{\prime}} P\right)} .
$$

where the overall normalization is computed in Appendix B and it differs from the one in [36] due to our conventions.

It is interesting to note that unlike (2.4) the one point function (2.7) does not exhibit "bulk poles" at imaginary $P$. Instead, it is zero at $P=-i n / \sqrt{\alpha^{\prime}}$. We interpreted the absence of poles as due to the fact that these D-branes are localized far from the bulk region of large and negative $\phi$. 


\section{D0-brane decay in $2 d$ string theory}

Let us consider the D0-brane which is the direct product of the localized Liouville brane times a Neumann boundary condition in time. The Liouville part contains only one Virasoro primary, the identity operator [36]. This is the boundary identity operator and it is normalizable since the brane is localized in the $\phi$ direction. It follows that the full physical open string spectrum contains only two states, one is the tachyon and the other is $\partial t$, the gauge field on the D-brane. This is a negative norm state which is responsible for imposing the constraint that, when we have $N$ D0-branes of this type, we should restrict to $U(N)$ invariant wave functions.

Now we can consider the boundary state which is a product of Sen's rolling tachyon boundary state for $t$ and the localized boundary state of [36] for $\phi$. We compute the closed string emission from this state as was done in [23]. We consider a decaying brane with open string tachyon $T=\lambda \cosh \left(t / \sqrt{\alpha^{\prime}}\right)$. We get that the decay rate to a massless bulk tachyons with momentum $P$ and energy $E=|P|$ is

$$
\mathcal{A}=\mathcal{A}_{t}(E) \mathcal{A}_{L}(P)
$$

where $\mathcal{A}_{L}$ was defined in (2.7) and the time part was computed in 23]

$$
\mathcal{A}_{t}=\frac{\pi e^{-i \sqrt{\alpha^{\prime}} E \log \hat{\lambda}}}{\sinh \left(\pi \sqrt{\alpha^{\prime}} E\right)}
$$

where $\hat{\lambda}=\sin \pi \tilde{\lambda}$ and $\tilde{\lambda}$ is the variable that appears in the description of the boundary state [1].

We finally get that the emission rate is

$$
\mathcal{A}=i 2 \sqrt{\pi} \frac{\sinh \left(\pi \sqrt{\alpha^{\prime}} P\right)}{\sinh \left(\pi \sqrt{\alpha^{\prime}} E\right)} e^{-i \sqrt{\alpha^{\prime}} E \log \hat{\lambda}} \mu^{-\frac{i}{2} \sqrt{\alpha^{\prime}} P} \frac{\Gamma\left(i \sqrt{\alpha^{\prime}} P\right)}{\Gamma\left(-i \sqrt{\alpha^{\prime}} P\right)}=2 \sqrt{\pi} i e^{-i \sqrt{\alpha^{\prime}} E \log \hat{\lambda}} e^{i \delta(P)}
$$

where the normalization is computed in the Appendix B. Remarkably, after we use the onshell condition $E=|P|$, we find that the amplitude is a constant times an energy dependent phase. ${ }^{\circ}$ As a result, we find that the rate for the number of particles $N=\int \frac{d P}{2 E}|\mathcal{A}|^{2}$ diverges logarithmically, and the expectation value for the emitted energy diverges linearly in the

3 A part of this phase, $e^{i \delta}(P) \equiv \mu^{-\frac{i}{2} \sqrt{\alpha^{\prime}} P} \frac{\Gamma\left(i \sqrt{\alpha^{\prime}} P\right)}{\Gamma\left(-i \sqrt{\alpha^{\prime}} P\right)}$ is the standard leg factor that appears for bulk Liouville theory operators (see, for instance, [41, [26]). We discuss this in appendix A. 
UV. This expression also has an IR divergence that is related to the fact that we are dealing with a massless field in $1+1$ dimensions.

The UV properties are identical to the ones found in [23 for the decay of D0 branes in 26-d bosonic string. In that case, the exponential decay of the amplitude was exactly cancelled by the exponentially growing density of closed string states. In the 2-d string there is no such growth in the density of states. But the coupling to the tachyon (3.3) does not decay exponentially. In fact it has a constant absolute value, leading to the same UV behavior of the particle production rate. This behavior of the amplitude is consistent with the divergence present in the open string channel when we consider a D-instanton array (the $\tilde{\lambda}=1 / 2$ states).

Note that the final state that is produced is a coherent state of the form

$$
|\psi\rangle \sim e^{\int_{0}^{\infty} \frac{d p}{\sqrt{2 E}} a_{p}^{\dagger} \mathcal{A}}|0\rangle
$$

up to an overall normalization factor, where $\mathcal{A}$ was given in (3.3).

We should end by saying that this does not agree with the closed string emission formula computed in 25], since [25] considered a D-brane extended in the Liouville direction.

\section{The $c=1$ Matrix Model}

Let us recall a few basic facts about the $c=1$ matrix model which describes twodimensional string theory. One starts with the Euclidean path integral for a Hermitian $N \times N$ matrix

$$
Z=\int D^{N^{2}} \Phi(x) \exp \left[-\beta \int_{-\infty}^{\infty} d x \operatorname{Tr}\left(\frac{1}{2}\left(D_{x} \Phi\right)^{2}+U(\Phi)\right)\right]
$$

where $x$ is the Euclidean time and $\beta$ is the inverse Planck constant. If we take the matrix potential to be $U=\frac{1}{2 \alpha^{\prime}} \Phi^{2}-\frac{1}{3} \Phi^{3}$ then the Feynman graphs may be thought of as discretized random surfaces embedded into one Euclidean dimension $x$. It can be shown [26] that $\alpha^{\prime}$ is the conventionally normalized inverse string tension parameter (this follows, for example, from the correct position of poles in the closed string amplitudes).

From the point of view of random surfaces, the matrix $\Phi$ is an auxiliary concept. Recently, a more direct physical interpretation of $\Phi$ was proposed [25]. According to [25], $\Phi$ is an open string tachyon field on D0-branes. We believe that the D0-branes which this field resides on are obtained from the localized boundary state for $\phi$ [36] multiplied by the 
Neumann boundary state for the time coordinate $t=i x$. It follows that $U(\Phi)$ should be thought of as the tachyon potential. Indeed, expanding the potential near the quadratic maximum, we find

$$
U(\Phi)=-\frac{1}{2 \alpha^{\prime}}\left(\Phi-\Phi_{0}\right)^{2}+O\left[\left(\Phi-\Phi_{0}\right)^{3}\right]
$$

The curvature of the potential at the maximum exactly agrees with the open string tachyon mass-squared $m_{T}^{2}=-1 / \alpha^{\prime}$, obtained as an on-shell condition on the vertex operator $e^{i E t}$. This serves as a consistency check on the identification of $\Phi$ with the tachyon field localized on the D0-branes of [36].

The open string spectrum also includes a non-dynamical gauge field $A$, corresponding to the vertex operator $\dot{t}$. It enters the covariant derivative in (4.1):

$$
D_{x} \Phi=\partial_{x} \Phi-[A, \Phi]
$$

$A$ acts as a lagrange multiplier that projects onto $S U(N)$ singlet wave functions.

The exact solvability of the model (4.1) in the singlet sector relies on the classic fact 29] that the $N$ eigenvalues of the matrix $\Phi$ act as free fermions and can hence be described by Slater determinant wave functions of $N$ variables. The ground state is obtained by filling the first $N$ levels to a Fermi level $-\mu_{F}$ (as measured from the local maximum of the potential). To take the double-scaling limit, one sends $\mu_{F} \rightarrow 0, \beta \rightarrow \infty$, keeping $\mu=\beta \mu_{F}$ fixed. The parameter $\mu$ is proportional to $1 / g_{s}$ and therefore has to be kept large in perturbation theory. The mass of the unstable D0-brane is the energy $\mu$ required to move an eigenvalue from the Fermi level to the top of the potential [25], and it scales correctly as $1 / g_{s}$.

The double-scaling limit zooms in on the local quadratic maximum of the potential. In this sense, the $c=1$ matrix model is equivalent to free fermions moving in an upside down quadratic potential. 4 Thus, we have a new example of exact large $N$ duality [25]. The $S U(N)$ symmetric matrix quantum mechanics (4.1) in an upside down harmonic oscillator potential, which exactly describes open strings on $N$ D0-branes of [36], is dual to Liouville theory coupled to $c=1$ matter which describes two-dimensional closed string theory together with its D0-branes.

We will be interested in a method for bosonizing the non-relativistic fermions. Several closely related ways of doing this are available in the literature [42,43]. We will find it

4 It is sometimes useful to keep in mind that the original potential includes a cutoff far from the maximum so that we have a finite number of levels. 
convenient to follow the formalism developed in [44,45] and reviewed in [26]. We briefly summarize this method.

The second quantized hamiltonian for a system of free fermions is

$$
\hat{H} \sim \int d y\left\{\frac{1}{2} \frac{\partial \Psi^{\dagger}}{\partial y} \frac{\partial \Psi}{\partial y}-\frac{y^{2}}{2 \alpha^{\prime}} \Psi^{\dagger} \Psi+\mu\left(\Psi^{\dagger} \Psi-N\right)\right\},
$$

where $\mu$ is the Lagrange multiplier necessary to fix the total number of fermions to equal $N$. As usual, it will be adjusted so as to equal the Fermi level of the $N$ fermion system. We may introduce new chiral fermionic variables $\Psi_{L}$ and $\Psi_{R}(t=i x$ is now the Lorentzian time),

$$
\Psi(y, t)=\frac{e^{i \mu t}}{\sqrt{2 v(y)}}\left[e^{-i \int^{y} d y^{\prime} v\left(y^{\prime}\right)+i \pi / 4} \Psi_{L}(y, t)+e^{i \int^{y} d y^{\prime} v\left(y^{\prime}\right)-i \pi / 4} \Psi_{R}(y, t)\right],
$$

where

$$
v(y)=\frac{d y}{d \tau}=\sqrt{\frac{y^{2}}{\alpha^{\prime}}-2 \mu}
$$

is the velocity of the classical trajectory of a particle at the Fermi level. In terms of the new variables, and using $\tau$ as the spatial coordinate, the Hamiltonian becomes

$$
\begin{aligned}
& \int d \tau\left[i \Psi_{R}^{\dagger} \partial_{\tau} \Psi_{R}-i \Psi_{L}^{\dagger} \partial_{\tau} \Psi_{L}+\frac{1}{2 v^{2}}\left(\partial_{\tau} \Psi_{L}^{\dagger} \partial_{\tau} \Psi_{L}+\partial_{\tau} \Psi_{R}^{\dagger} \partial_{\tau} \Psi_{R}\right)\right. \\
& \left.+\frac{1}{4}\left(\Psi_{L}^{\dagger} \Psi_{L}+\Psi_{R}^{\dagger} \Psi_{R}\right)\left(\frac{v^{\prime \prime}}{v^{3}}-\frac{5\left(v^{\prime}\right)^{2}}{2 v^{4}}\right)\right]
\end{aligned}
$$

where $v^{\prime} \equiv d v / d \tau$. We notice that for large $\tau$ (and hence large $v$ ) the hamiltonian is approximately relativistic. This large $\tau$ region will be our primary concern since we are interested in translating to bosonic variables in the asymptotic future and past. If we

5 One may be concerned about the region near the turning point of the classical trajectory where there are singularities in the corrections to non-relativistic terms. A way to deal with this was suggested in 26,28]: instead of wave functions in position space, we could consider them in momentum space. Due to the special nature of the upside down harmonic oscillator potential, the hamiltonian preserves its form, except now the Fermi level is above the barrier. After this transformation, the velocity $v(\tau)=\sqrt{2 \mu} \cosh \left(\tau / \sqrt{\alpha^{\prime}}\right)$ has no zeroes and the range of $\tau$ may be taken from $-\infty$ to $\infty$. In this momentum space picture a single branch of Fermi surface has signals of only one chirality and there are no end-point singularities. Another way to think about this picture is to formally send $\mu \rightarrow-\mu$ in the original problem so that the Fermi level is above the top of the potential [26]. Then the D-brane is a hole in the Fermi sea at precisely the zero energy. Our primary concern will be the region far from the singularity where either the momentum space or position space formulation is non-singular. 
introduce a cutoff, then the energy levels are approximately given by

$$
\epsilon_{n} \sim-\mu+n / T+\frac{\delta^{\prime}(n / T, \mu)}{T^{2}}+\cdots
$$

where $2 \pi T=\oint \frac{d y}{v(y)}=\oint d \tau$, and $\delta^{\prime}$ is related to the derivative of the reflection amplitude from the inverted harmonic oscillator barrier and from the cutoff barrier. We will be interested in the limit of large $T$ so the the last term in (4.8) does not contribute. It is important to notice that (4.8) is valid even if $\epsilon_{n} \sim \mu$. So we dropped the second term in (4.8) in the limit $T \rightarrow \infty$, even though it diverges when $\epsilon_{n} \rightarrow 0$ (top of the potential). So, then we can write the left-moving fermion as in 44,45, 26]:

$$
\Psi_{L}(\tau, t) \sim \int_{0}^{\infty} d p e^{i p(\tau+t)} b_{p}^{\dagger}+\int_{-\infty}^{0} d p e^{i p(\tau+t)} d_{p}
$$

in the region where $y \gg 1, \tau \gg 1$. Since here $v \gg 1$, the fermions are approximately relativistic. We concentrate on the left moving fermion. There is a similar expression for the right moving fermions.

The relativistic fermion $\Psi_{L}$ is bosonized as

$$
\Psi_{L}(\tau+t)|0\rangle=e^{i 2 \sqrt{\pi} \phi_{L}(\tau+t)} \mid 0>
$$

where $\phi$ is a canonically normalized massless scalar field. $\phi$ is linearly related to the bulk tachyon field $V$. The relation is non-local. In Fourier space the relation is just a momentum dependent phase,

$$
\phi_{p}=e^{i \delta(p)} V_{p}
$$

This phase is discussed in more detail in Appendix A. It has not been fully derived, as far as we know, but its presence can be indirectly seen by looking at the Euclidean theory. It is necessary to include it when we compare the free fermion amplitudes to the string theory amplitudes.

\subsection{D-brane decay in the matrix model}

If we start with a single fermion near the top of the potential this fermion will roll down and go to infinity. When it is in the large $\tau$ region it becomes relativistic and we can bosonize it using the formulae in (4.10). Note then that the state (4.10) agrees precisely with the form of the coherent state that we get at tree level in string theory (3.4). However this is not the precise description. In order to obtain a precise description we should notice 
that the free fermion will have some particular wavefunction $\psi(\tau+t)$. This wavefunction is the one that results from localizing the eigenvalue on top of the potential. So a more precise description of the final state would be to write it as

$$
\int d \tau \psi(\tau) \Psi_{L}(\tau)|0\rangle=\int d \tau \psi(\tau) e^{i 2 \sqrt{\pi} \int \frac{d p}{2 \pi \sqrt{2 E}} e^{-i p \tau} a_{p}^{\dagger}}|0\rangle
$$

Here $\psi$ is the wavefunction that results from taking a wavefunction that is initially localized near the top and evolving it to the asymptotic region and rexpressing it in terms of the relativistic fermions.

In particular, a single fermion of energy $E$ is equivalent to

$$
\int d \tau e^{i E \tau} \Psi_{L}(\tau)|0\rangle=\int d \tau e^{i E \tau} e^{i 2 \sqrt{\pi} \int \frac{d p}{2 \pi \sqrt{2 E}} e^{-i p \tau} a_{p}^{\dagger}}|0\rangle
$$

where $a_{p}$ is the massless boson annihilation operator. Note that this state precisely agrees with the state that we found above (3.4), except for the projection onto a definite energy state.

A more precise discussion has to take into account the fact that this fermion has to be anti-symmetrized with the fermions forming the fermi sea. For large $\mu$ these effects are very small. The full specification of the initial state involves also saying what the fermions forming the fermi sea are doing. If we leave them untouched when we add the extra eigenvalue we note that the matrix model expressions for the closed string emission agree with the expressions obtained in [23] with the so called "Hartle-Hawking" contour, which was the one naturally related to the Euclidean computation. One surprising consequence of this contour choice is that at tree level the closed string state seemed to be independent (up to an overall time delay) of $\tilde{\lambda}$. We see that this dependence will come in when we include the wavefunction of the initial state, as in (4.12).

Sen's boundary state with parameter $\tilde{\lambda}$ [1] corresponds, in the matrix model, to an eigenvalue that starts at the position

$$
y=-\sqrt{2 \alpha^{\prime} \mu} \sin \pi \tilde{\lambda}
$$

Then the energy of the state is given by $E=\mu \cos ^{2}(\pi \tilde{\lambda})$, as in [1]. The time delay in the classical evolution of this trajectory relative to the classical trajectory at the Fermi level is

$$
\Delta t=\log (\sin \pi \tilde{\lambda})
$$

It is easy to check that this is precisely the time delay that appears as a phase in (3.2). 


\subsection{D-branes in the Euclidean 2d String}

Let us also try to give matrix model interpretation to D-branes of the Euclidean 2-d string theory localized at large $\phi$. Putting the Neumann boundary condition on the field $X$ once again corresponds to placing an eigenvalue at the top of the upside down potential. It is well-known [4,5] that one can turn on an exactly marginal boundary operator

$$
\lambda \oint d \sigma \cos \left(X / \sqrt{\alpha^{\prime}}\right)
$$

which interpolates between the Neumann and the Dirichlet boundary conditions on $X$. We believe that turning on this potential corresponds to an eigenvalue in the matrix model executing classical Euclidean motion in the forbidden region

$$
y(x)=\sqrt{2 \alpha^{\prime} \mu} \sin \pi \tilde{\lambda} \cos \left(x / \sqrt{\alpha^{\prime}}\right) .
$$

The value $\tilde{\lambda}=1 / 2$, corresponding to the Dirichlet boundary condition on $X$, is dual to the eigenvalue trajectory at the Fermi level. Thus, the D-instanton is indeed related to the eigenvalue tunnelling at the Fermi level, as speculated in [32].

There is an interesting subtlety, however. In the matrix model the energy of an eigenvalue at the maximum is $\mu$, while the action of the tunnelling trajectory is $\pi \mu \sqrt{\alpha^{\prime}}$. The tunnelling trajectory has to be interpreted as 'half' of a D-instanton. 6 The D-instanton corresponds to tunnelling from the filled region to the unfilled, and then tunnelling back (i.e. the bounce). Then the D-instanton action $2 \pi \mu \sqrt{\alpha^{\prime}}$ is related to the tension of the D0 brane by

$$
\frac{T_{-1}}{T_{0}}=2 \pi \sqrt{\alpha^{\prime}}
$$

This agrees with the descent relation following from the boundary state formalism. This relation is completely determined by the Neumann and Dirichlet boundary states of the field $X$ and does not involve the knowledge of the Liouville boundary state.

\section{Discussion}

Notice that the divergences in the energy and norm of the state that we had in the tree level result (3.3) are absent from the all orders result (4.12). We also clearly see that the total energy emitted is obviously equal to the energy of the initial state characterized

\footnotetext{
${ }^{6}$ For a recent discussion of half-instantons and merons, see [39].
} 
by $\psi$. In this example the divergence of the tree level answer is regularized by considering the quantum mechanics of the tachyon field. So it looks like a one loop effect in the open string channel. In this case the fermions are completely free, so it is hard to extract a general lesson for the 26 dimensional bosonic string. One lesson is that taking into account the quantum mechanics of the open strings is crucial for finding a regulated expression. Another general lesson is that the wavefunction of the open string modes gets imprinted in the final wave function for the closed strings. In this completely solvable example we find that the "tachyon matter" state introduced in [1] is just the state of closed strings that the brane decays into.

Another interesting issue is the relation between the tachyon potentials discussed in the context of string field theory [46,47,48] to the simple inverted quadratic potential we find here. We think that the relation is the following. The string field theory potential is only part of the parabola. It is the region above the fermi sea on the left side and the whole parabola on the right side (the unfilled side). This agrees with two qualitative features. One is that at a finite distance there is a minimum corresponding to the closed string vacuum, this corresponds to placing an eigenvalue at the fermi surface. On the "wrong" side the potential is unbounded below.

Let us also comment on the D1-branes obtained from the extended Liouville boundary state of [34,35] multiplied by the Neumann boundary state for the (Euclidean) time. We believe that a dual large $N$ description of the 2-d string theory in presence of such a space-time filling brane is given by

$$
\begin{gathered}
Z=\int D^{N^{2}} \Phi(x) D^{2 N} V(x) e^{-\beta S} \\
S=\int_{-\infty}^{\infty} d x\left[\operatorname{Tr}\left(\frac{1}{2}\left(D_{x} \Phi\right)^{2}+U(\Phi)\right)+\frac{1}{2}\left(D_{x} V\right)^{\dagger} D_{x} V+\frac{1}{2} m^{2} V^{\dagger} V+g V^{\dagger} \Phi V\right],
\end{gathered}
$$

where $V$ is a complex vector. Integrating over $V$ inserts into random surfaces dynamical boundaries that can wander in the time direction (models of this type were considered in [49,50,51). By tuning $m^{2}$ and $g$ together with $\beta$, we expect to find a scaling model with independent parameters $\mu_{b}$ and $\mu$ as in (2.5). The massless open string 'tachyon' is described by operators $\int d x e^{i q x} V^{\dagger} V(x)$.

If we consider a model without the kinetic term for $V$, then integration over $V$ generates boundaries localized in the time direction, with subsequent averaging over possible locations of the boundary. Such boundaries with Dirichlet boundary conditions on the time coordinate are the macroscopic loops which were studied in [52,41]. 
We should also emphasize another important distinction between the D0-brane and the macroscopic loop. The former is localized in $\phi$, while the latter extends all the way to the spatial boundary at $\phi \rightarrow-\infty$. Therefore, the macroscopic loop is an observable in the theory which is associated with a change in the boundary conditions. It differs from the localized D0-brane which corresponds to a dynamical excitation of the system.

It is important to distinguish the eigenvalue $y$ from the Liouville coordinate $\phi$. The spaces parameterized by them are related to each other through a nonlocal transform 41,53. The time-dependence of an eigenvalue (the open string tachyon) does not describe motion of a D0-brane in $\phi$; rather, it describes the decay of a D0-brane localized at large $\phi$ [1]. The time dependence in the $\phi$ description arises only from the boundary interaction (1.1).

\section{Acknowledgments}

We would like to thank N. Itzhaki, J. McGreevy, A.M. Polyakov and H. Verlinde for useful discussions. The research of JM and NS is supported in part by DOE grant DEFG02-90ER40542. The research of IRK was supported in part by the National Science Foundation Grants No. PHY-9802484 and PHY-0243680. Any opinions, findings, and conclusions or recommendations expressed in this material are those of the authors and do not necessarily reflect the views of the National Science Foundation.

\section{Appendix A. Leg Factors}

In this appendix we discuss a bit more the phase (4.11) present in the relation between the string theory computations and the matrix model computations. These leg factors are typical in relations between closed string amplitudes and their origin was discussed, for

example, in 41,26]. Let us first discuss the Euclidean matrix model. It has finite boundary operator (whose $l \rightarrow 0$ limit is the puncture operator)

$$
O(l, q)=\int d x e^{i q x} \operatorname{Tr} e^{-l \Phi(x)}
$$

which is translated into

$$
\frac{1}{2} \int d x e^{i q x} \int_{0}^{\infty} d \tau e^{-l y(\tau)}: \Psi_{L}^{\dagger} \Psi_{L}+\Psi_{R}^{\dagger} \Psi_{R}(\tau, x):
$$


where $y(\tau)$ is the classical trajectory at the Fermi level. Upon bosonization, we find

$$
O(l, q) \sim \int d x e^{i q x} \int d \tau e^{-l y(\tau)} \partial_{\tau} X \sim i \int_{-\infty}^{\infty} d k F(k, l) k \tilde{X}(q, k)
$$

where

$$
F(k, l)=\int_{0}^{\infty} d \tau e^{-l \sqrt{2 \alpha^{\prime} \mu} \cosh \left(\alpha^{\prime} \tau\right)} \cos (k \tau) .
$$

In working with the Euclidean matrix model, there is freedom in multiplying puncture operator $O(l \rightarrow 0, q)$ corresponding to tachyon of momentum $q$ by a smooth function of $q$ [26]. The normalization may be chosen in such a way that, in the correlation function, each operator is accompanied by a leg factor containing all the poles due to the discrete states:

$$
\mu^{\sqrt{\alpha^{\prime}}|q| / 2} \frac{\Gamma\left(-\sqrt{\alpha^{\prime}}|q|\right)}{\Gamma\left(\sqrt{\alpha^{\prime}}|q|\right)} .
$$

We have picked the numerator so that it reproduces the poles present in the relation. The special property of this choice of normalization is that after continuation to Lorentzian

signature, $X \rightarrow i t$, the leg factor corresponding to operator $e^{ \pm i P t} e^{\left(2 \alpha^{\prime-1 / 2}+i P\right) \phi}$ becomes a pure phase

$$
\mu^{-\frac{i}{2} \sqrt{\alpha^{\prime}} P} \frac{\Gamma\left(i \sqrt{\alpha^{\prime}} P\right)}{\Gamma\left(-i \sqrt{\alpha^{\prime}} P\right)} .
$$

These phases are indeed present in all Liouville theory calculations and, in particular, in the disk 1-point function (3.3). However, these phases do not appear in the S-matrix obtained using Lorentzian semiclassical free fermions with Polchinski's methods [28]. Therefore, to compare free fermion calculations with Liouville theory, the phases have to be added "by hand." As we explained above, one justification for these phases is to start in the Euclidean signature, where the leg factors are certain observable (they contain poles due to the discrete states), and later continue to Lorentzian signature.

\section{Appendix B. Matching of coefficients}

In this Appendix we match the coefficients between the free fermion computation and the Liouville computation.

Let us start with the matrix model answer

$$
\Psi_{L}|0\rangle=e^{i 2 \sqrt{\pi} \phi_{L}}|0\rangle=e^{i 2 \sqrt{\pi} \int_{0}^{\infty} \frac{d p}{2 \pi} \frac{1}{\sqrt{2 E}} a_{p}^{\dagger}}|0\rangle
$$


where $\phi$ is a canonically normalized scalar field, and similarly the $a_{p}^{\dagger}$ are the creation operators canonically normalized, with the usual continuum normalization $\left(\left[a_{p}, a_{p^{\prime}}^{\dagger}\right]=\right.$ $\left.2 \pi \delta\left(p-p^{\prime}\right)\right)$. In the string theory we find that the amplitude for emitting on shell strings is given by

$$
\mathcal{A}=\mathcal{N} e^{i \delta}
$$

where $e^{i \delta}$ is an unimportant phase. Our goal is to show that the normalization factor is $\mathcal{N}=2 \sqrt{\pi}$ in agreement with the matrix model.

For this purpose we note that formally the emission amplitude is related to the imaginary part of the one loop Feynman amplitude through

$$
2 \operatorname{Im}\left(Z_{1}\right)=\int_{0}^{\infty} \frac{d p}{2 \pi} \frac{|\mathcal{A}|^{2}}{2 E(p)}
$$

We start with the one loop amplitude for open strings for the brane with Neuman boundary conditions in the time direction. Using the character for the $\chi_{(1,1)}$ representation of [36], this is given by

$$
Z_{1}=\int_{0}^{\infty} \frac{d t}{2 t} \operatorname{Tr}_{\text {open }} e^{-2 \pi t L_{0}}=V_{t} \int_{0}^{\infty} \frac{d t}{2 t} \int_{-\infty}^{\infty} \frac{d k_{0}}{2 \pi} e^{-2 \pi t \alpha^{\prime} k_{0}^{2}}\left(e^{+2 \pi t}-1\right)
$$

where $V_{t}$ is the length of the time direction. This partition function shows only two open string states, the tachyon and the $A_{0}$ gauge constraint (which gives the -1 ). We rewrite it in the closed string channel as $\left(\alpha^{\prime}=1\right)$

$$
Z_{1}=V_{t} \int_{0}^{\infty} d s \int_{0}^{\infty} \frac{d p}{2 \pi}(\sinh \pi p)^{2} e^{-2 \pi s \frac{p^{2}}{4}}
$$

The momentum integral runs over half the real axis because the closed string states labeled by $p$ and $-p$ are the same (up to a phase which is the reflection amplitude). If we consider instead the decaying brane we should substitute [23]

$$
V_{t}=\int \frac{d k_{0}}{2 \pi}\left(2 \pi \delta\left(k_{0}\right)\right)^{2} \rightarrow \int \frac{d k_{0}}{2 \pi}\left(\frac{\pi}{\sinh \pi k_{0}}\right)^{2} e^{-2 \pi s \alpha^{\prime} \frac{-k_{0}^{2}}{4}}
$$

Inserting this in (B.5) and (formally) integrating over $s$ find that we get the expression

$$
Z_{1}=\frac{2}{\pi} \int_{0}^{\infty} \frac{d p}{2 \pi} \int_{-\infty}^{\infty} \frac{d k_{0}}{2 \pi}\left(\frac{\pi \sinh \pi p}{\sinh \pi k_{0}}\right)^{2} \frac{1}{-k_{0}^{2}+p^{2}-i \epsilon}
$$

The imaginary part comes from the two poles at $k_{0}= \pm p$ and gives

So we find

$$
\operatorname{Im}\left[\int \frac{d k_{0}}{2 \pi} \frac{1}{-k_{0}^{2}+p^{2}-i \epsilon} f\left(k_{0}\right)\right]=\frac{f(E(p))}{2 E(p)} ; \quad E(p)=p
$$

$$
2 \operatorname{Im}\left(Z_{1}\right)=4 \pi \int_{0}^{\infty} \frac{d p}{2 \pi} \frac{1}{2 E(p)}
$$

Comparing this with (B.3) we find that

$$
\mathcal{A}=2 \sqrt{\pi} e^{i \delta}
$$




\section{References}

[1] A. Sen, "Rolling tachyon," JHEP 0204, 048 (2002) arXiv:hep-th/0203211].

[2] A. Sen, "Tachyon matter," JHEP 0207, 065 (2002) arXiv:hep-th/0203265.

[3] A. Sen, "Field theory of tachyon matter," Mod. Phys. Lett. A 17, 1797 (2002) arXiv:hep-th/0204143.

[4] C. G. Callan, I. R. Klebanov, A. W. Ludwig and J. M. Maldacena, "Exact solution of a boundary conformal field theory," Nucl. Phys. B 422, 417 (1994) arXiv:hepth/9402113].

[5] J. Polchinski and L. Thorlacius, "Free Fermion Representation Of A Boundary Conformal Field Theory," Phys. Rev. D 50, 622 (1994) arXiv:hep-th/9404008.

[6] M. Gutperle and A. Strominger, "Spacelike branes," JHEP 0204, 018 (2002) arXiv:hep-th/0202210.

[7] A. Sen, "Time evolution in open string theory," JHEP 0210, 003 (2002) arXiv:hepth/0207105.

[8] P. Mukhopadhyay and A. Sen, "Decay of unstable D-branes with electric field," JHEP 0211, 047 (2002) arXiv:hep-th/0208142.

[9] A. Strominger, "Open string creation by S-branes," arXiv:hep-th/0209090.

[10] A. Sen, "Time and tachyon," arXiv:hep-th/0209122.

[11] F. Larsen, A. Naqvi and S. Terashima, "Rolling tachyons and decaying branes," arXiv:hep-th/0212248.

[12] M. Gutperle and A. Strominger, "Timelike Boundary Liouville Theory," arXiv:hepth/0301038].

[13] A. Maloney, A. Strominger and X. Yin, "S-brane thermodynamics," arXiv:hepth/0302146].

[14] B. Chen, M. Li and F. L. Lin, "Gravitational radiation of rolling tachyon," JHEP 0211, 050 (2002) arXiv:hep-th/0209222.

[15] S. J. Rey and S. Sugimoto, "Rolling Tachyon with Electric and Magnetic Fields T-duality approach," arXiv:hep-th/0301049.

[16] N. Moeller and B. Zwiebach, "Dynamics with infinitely many time derivatives and rolling tachyons," JHEP 0210, 034 (2002) arXiv:hep-th/0207107.

[17] S. Sugimoto and S. Terashima, "Tachyon matter in boundary string field theory," JHEP 0207, 025 (2002) arXiv:hep-th/0205085.

[18] J. A. Minahan, "Rolling the tachyon in super BSFT," JHEP 0207, 030 (2002) arXiv:hep-th/0205098.

[19] J. Kluson, "Time dependent solution in open Bosonic string field theory," arXiv:hepth/0208028; "Exact solutions in open Bosonic string field theory and marginal deformation in CFT," arXiv:hep-th/0209255. 
[20] T. Okuda and S. Sugimoto, "Coupling of rolling tachyon to closed strings," Nucl. Phys. B 647, 101 (2002) [arXiv:hep-th/0208196].

[21] I. Y. Aref'eva, L. V. Joukovskaya and A. S. Koshelev, "Time evolution in superstring field theory on non-BPS brane. I: Rolling tachyon and energy-momentum conservation," arXiv:hep-th/0301137.

[22] A. Ishida and S. Uehara, "Rolling down to D-brane and tachyon matter," JHEP 0302, 050 (2003) arXiv:hep-th/0301179.

[23] N. Lambert, H. Liu and J. Maldacena, "Closed strings from decaying D-branes," arXiv:hep-th/0303139.

[24] D. Gaiotto, N. Itzhaki and L. Rastelli, "Closed strings as imaginary D-branes," arXiv:hep-th/0304192.

[25] J. McGreevy and H. Verlinde, "Strings from tachyons: The c $=1$ matrix reloaded," arXiv:hep-th/0304224.

[26] I. R. Klebanov, "String theory in two-dimensions," arXiv:hep-th/9108019.

[27] P. Ginsparg and G. W. Moore, "Lectures On 2-D Gravity And 2-D String Theory," arXiv:hep-th/9304011.

[28] J. Polchinski, "What is String Theory?" arXiv:hep-th/9411028

[29] E. Brezin, C. Itzykson, G. Parisi and J. Zuber, CMP 59 (1978) 35.

[30] S. H. Shenker, "The Strength Of Nonperturbative Effects In String Theory," RU-9047 Presented at the Cargese Workshop on Random Surfaces, Quantum Gravity and Strings, Cargese, France, May 28 - Jun 1, 1990

[31] A. Dhar, G. Mandal and S. R. Wadia, "A Time dependent classical solution of c $=1$ string field theory and nonperturbative effects," Int. J. Mod. Phys. A 8, 3811 (1993) arXiv:hep-th/9212027.

[32] J. Polchinski, "Combinatorics Of Boundaries In String Theory," Phys. Rev. D 50, 6041 (1994) arXiv:hep-th/9407031.

[33] M. B. Green, "A Gas of D instantons," Phys. Lett. B 354, 271 (1995) arXiv:hepth/9504108].

[34] V. Fateev, A. B. Zamolodchikov and A. B. Zamolodchikov, "Boundary Liouville field theory. I: Boundary state and boundary two-point function," arXiv:hep-th/0001012.

[35] J. Teschner, "Remarks on Liouville theory with boundary," arXiv:hep-th/0009138.

[36] A. B. Zamolodchikov and A. B. Zamolodchikov, "Liouville field theory on a pseudosphere," arXiv:hep-th/0101152.

[37] A. M. Polyakov, "Gauge fields and space-time," Int. J. Mod. Phys. A 17S1, 119 (2002) arXiv:hep-th/0110196.

[38] J. A. Harvey, P. Horava and P. Kraus, "D-sphalerons and the topology of string configuration space," JHEP 0003, 021 (2000) arXiv:hep-th/0001143.

[39] N. Drukker, D. J. Gross and N. Itzhaki, "Sphalerons, merons and unstable branes in AdS," Phys. Rev. D 62, 086007 (2000) arXiv:hep-th/0004131. 
[40] B. Ponsot and J. Teschner, "Boundary Liouville field theory: Boundary three point function," Nucl. Phys. B 622, 309 (2002) arXiv:hep-th/0110244.

[41] G. W. Moore and N. Seiberg, "From loops to fields in 2-D quantum gravity," Int. J. Mod. Phys. A 7, 2601 (1992).

[42] S. R. Das and A. Jevicki, "String Field Theory And Physical Interpretation Of D = 1 Strings," Mod. Phys. Lett. A 5, 1639 (1990).

[43] J. Polchinski, "Classical Limit Of (1+1)-Dimensional String Theory," Nucl. Phys. B 362, 125 (1991).

[44] A. M. Sengupta and S. R. Wadia, "Excitations And Interactions In D = 1 String Theory," Int. J. Mod. Phys. A 6, 1961 (1991).

[45] D. J. Gross and I. R. Klebanov, "Fermionic String Field Theory Of C $=1$ TwoDimensional Quantum Gravity," Nucl. Phys. B 352, 671 (1991).

[46] A. Sen and B. Zwiebach, "Tachyon condensation in string field theory," JHEP 0003, 002 (2000) arXiv:hep-th/9912249.

[47] A. A. Gerasimov and S. L. Shatashvili, "On exact tachyon potential in open string field theory," JHEP 0010, 034 (2000) arXiv:hep-th/0009103.

[48] D. Kutasov, M. Marino and G. W. Moore, "Some exact results on tachyon condensation in string field theory," JHEP 0010, 045 (2000) arXiv:hep-th/0009148].

[49] Z. Yang, "Dynamical Loops In D = 1 Random Matrix Models," Phys. Lett. B 257, 40 (1991).

[50] J. A. Minahan, "Matrix models and one-dimensional open string theory," Int. J. Mod. Phys. A 8, 3599 (1993) arXiv:hep-th/9204013.

[51] V. A. Kazakov and I. K. Kostov, "Loop gas model for open strings," Nucl. Phys. B 386, 520 (1992) arXiv:hep-th/9205059.

[52] G. W. Moore, N. Seiberg and M. Staudacher, "From loops to states in 2-D quantum gravity," Nucl. Phys. B 362, 665 (1991).

[53] J. Polchinski, "On the nonperturbative consistency of $d=2$ string theory," Phys. Rev. Lett. 74, 638 (1995) arXiv:hep-th/9409168. 